\title{
Study of the transportation behavior of nanoparticles through low-porosity sand pack in the absence and presence of oil
}

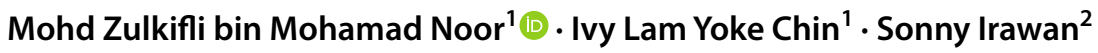

Received: 3 October 2018 / Accepted: 9 April 2019 / Published online: 16 April 2019

(c) The Author(s) 2019

\begin{abstract}
Nanoparticles exhibit unique physical properties and chemical properties and hence have received much attention from scientists and researchers in different areas of biological sciences. Nanoparticles are employed in a wide range of applications causing large quantities of these materials to be released into the environment. Yet, issues related to how and where these particles are released into the porous media still remain as major challenges. The objective of this research is directed toward the study of oxide nanoparticles transport in low-porosity sand pack and determine the distance transport by the nanoparticles in the absence and presence of oil. The nanopowders were dispersed in de-ionized water, and the horizontal column was packed with low-porosity sand (30-40\% porosity) of the size of $500 \mu \mathrm{m}$. This experiment was carried out with four different pore volume of nanoparticle suspensions ranging from 0.25 to $1.0 \mathrm{PV}$. The column effluents were analyzed using atomic absorption spectroscopy to determine the morphology of the elements existed in the effluents. The resistivity of four different sections (metal rod 1,2, metal rod 2,3, metal rod 3,4 and metal rod 1,4) were measured using a multimeter. The transport of nanoparticles was the smoothest in paraffin oil, followed by water. In the absence of paraffin oil, the conductivity and resistivity value in water were $0.022 \mathrm{~S} / \mathrm{m}$ and $44.90 \Omega \mathrm{m}$, respectively, after 1.0 PV injection. In the presence of paraffin oil, the conductivity and resistivity reading were $0.026 \mathrm{~S} / \mathrm{m}$ and $38.66 \Omega \mathrm{m}$, respectively, after 1.0 PV injection. This indicated that the transport of nanoparticles in the presence of oil had lower resistance compared to the resistance in the absence of oil and thus, the distance transported by nanoparticles in the presence of oil was longer compared to the distance transported in the absence of oil.
\end{abstract}

Keywords Oxide nanoparticle transport $\cdot$ Low-porosity sand $\cdot$ Conductivity $\cdot$ Resistivity

\section{Introduction}

Nanomaterials are employed in a variety of applications, from articles of daily use to spaceships. Recently, nanoparticles have been used for the remediation of contaminated groundwater. Nanoparticles released into the environment move to groundwater and surface water through soil layers. The travel of nanoparticles through the environment can be hazardous to humans and the environment. Movement of nanoparticles depends on the physical and chemical

Mohd Zulkifli bin Mohamad Noor

mzulkifli@ump.edu.my

1 Faculty of Chemical and Natural Resources Engineering, Universiti Malaysia Pahang, 26300 Gambang, Pahang, Malaysia

2 Department of Petroleum Engineering, Universiti Teknologi PETRONAS, 32610 Bandar Seri Iskandar, Perak, Malaysia conditions of the environment. Although nanomaterial hazards have been much researched recently, the transport and fate of nanomaterials in the environment have not received sufficient attention (Jeong 2009). The projections of a rising load of nanoparticles into the environment have raised concerns about their dispersion, as well as their potential toxic effect on specific organisms and on entire ecosystems. Nanoparticles are highly reactive and toxic to aquatic organisms as they are small enough to cross cell membranes. Therefore, suitable understanding of their fate and transport in the environment is required to assess the potential risks these particles pose to public and environmental health (Wang 2012). However, only a limited amount of investigations had been conducted to study the environmental fate and transport of nanoparticles. The transport of nanoparticles needs to be tested and validated before the nanoparticles can be fully utilized as it is a new application in enhance oil recovery (Bayat 2015). Besides, recent studies have shown that

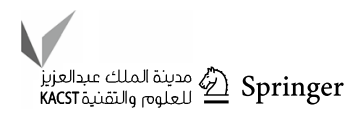


the small dimensions and enhanced reactivity of nanoparticles may cause risks to human health and this potentially irresistible effects of nanoparticles on the environment are just starting to be recognized. Because of the concern over potential threats of nanoparticle releases into the soil-water environment, a study should be carried out to investigate the transport, retention and adsorption of nanoparticles in saturated porous media (Hoet 2004).

\section{Materials and methods}

\section{Materials}

The sand will be collected from Teluk Cempedak, Kuantan and sieved using sieve with the size of $500 \mu \mathrm{m}$. The nanoparticles powder used in this study which is iron(III) oxide nanopowder will be obtained from the established supplier. The paraffin oil will be obtained from analytical laboratory UMP.

\section{Nanoparticles suspensions preparation}

The concentration of nanoparticles suspension used in this study is $80 \mathrm{mg} / \mathrm{L}$. The preparation of nanoparticles suspensions starts with the weighing of $0.008 \mathrm{~g}$ of nanoparticles powder in a weighing boat using a digital weight balance. After that, the nanopowder will be added to $1 \mathrm{~L}$ of de-ionized water (DIW) to form nanofluids. The reason for selecting DIW as the dispersion medium is to avoid the effects of solution chemistry (i.e., ionic strength and $\mathrm{pH}$ ) on the nanoparticles' stability and transport. After that, the mixture will be stirred using magnetic stirrer at ambient temperature for $1 \mathrm{~h}$. The mixture will be then ultrasonicated by an ultrasonic bath for a period of $6 \mathrm{~h}$ to obtain homogenous suspensions. The magnetic stirring helps to disperse the nanopowders evenly in the base fluid, but the energy is not enough to break any agglomeration of nanoparticles. So, an ultrasonic bath is used to break the agglomerations of nanoparticles.

\section{Porous media preparation}

The columns are constructed using clear polyvinyl chloride pipe (PVC) to allow direct observations during the sample preparation and measurement process. The length of the column is $35 \mathrm{~cm}$ with an inner diameter of $6.6 \mathrm{~cm}$. Columns will be packed with sand slowly. After each filling, the column was tapped to eliminate air bubbles and agitate to promote compaction of the sand (Bradford 2012). Agitating the samples helps to reduce the porosity resulting from grain stacking. The porosity of the columns is estimated to be $30-40 \%$. To prevent the sand particles to flow out with the solutions, two fine mesh wires were put at the inlet and outlet of the column. Four metal screws are placed on the column in order to ease the measurement of resistivity.

\section{Experimental setup and operation}

A schematic representation of the experimental set up used in this study is shown in Fig. 1.

Firstly, $250 \mathrm{~mL}$ of water will be measured and transferred to a $250 \mathrm{~mL}$ measuring cylinder to be introduced into the column using a peristaltic pump with volumetric flow rate of $45 \mathrm{~mL} / \mathrm{min}$ and the volume of water collected at the outlet of the column will be recorded. A beaker will be placed at the outlet of the column to collect the effluent of the column. This step is repeated for three to five times until the volume of water at the inlet and the outlet is the same. The total volume of water introduced into the column is recorded. The porosity of sand pack is calculated. After that, $0.25 \mathrm{PV}$ of nanoparticles suspensions is introduced into the column using peristaltic pump and the electrical resistivity of four different sections (metal rod 1,2, metal rod 2,3, metal rod 3,4 and metal rod 1,4) are measured using a digital multimeter. The readings of multimeter are recorded after the fluctuations stop during the measurement to avoid random errors. The sample effluent is collected at the outlet of the column. These steps are repeated by introducing different pore volumes of nanoparticles suspensions $(0.5 \mathrm{PV}, 0.75 \mathrm{PV}$

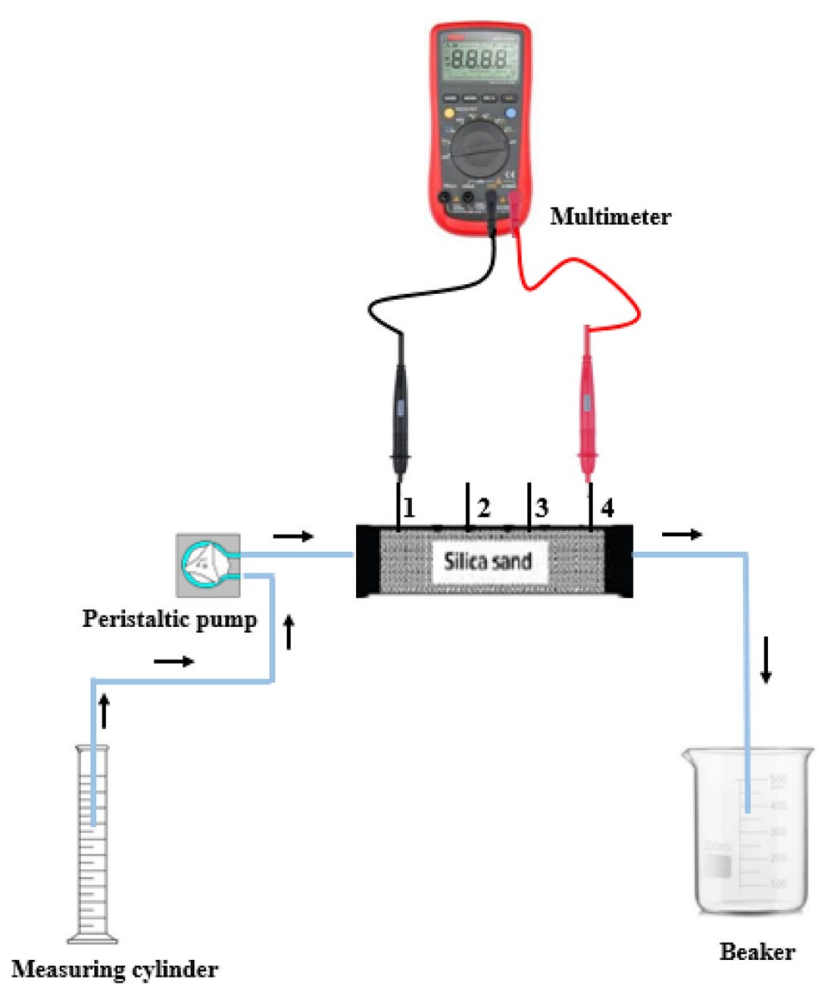

Fig. 1 Schematic diagram of experimental setup 
and 1.0 PV). The sample effluent collected will be analyzed using atomic absorption spectroscopy (AAS).

The experiment is conducted at room temperature. According to Caldelas (2011), the effect of temperature on NP retention is insignificant due to weak temperature dependence for the adsorption and desorption of NPs. By contrast, Guan et al. prepared iron oxide nanoparticles at different temperatures and the results showed that the NP at higher temperature has larger particle size due to agglomeration. The mechanism of temperature effect is complex and difficult to explain since it involves several variables. Increasing temperature tends to decrease the zeta potential of the particles which indicates the decrease in nanofluid stability and this would result in agglomeration of nanoparticles (Agista 2018). Further study on the temperature effect should be performed to obtain a better understanding of its effect on the transportation of oxide nanoparticles.

\section{Atomic absorption spectroscopy (AAS) analysis}

Atomic absorption can be defined as the absorption of light by activated atoms. Such absorption occurs on very narrow spectral lines, the so-called absorption or resonance lines (Zettner 1964). The operation of an atomic absorption spectrophotometer can be explained based on the following principle. The wavelength at which light is absorbed is specific for each element. If monochromatic light of a specific wavelength is provided, it will be absorbed only by atoms of that element whose resonance line is identical with the wavelength of the light source and not by any others. The amount of light absorbed at this wavelength will increase as the number of atoms of the selected element in the light path increases, and is proportional to the concentration of absorbing atoms. The relationship between the amount of light absorbed and the concentration of the analyte present in known standards can be used to determine unknown concentrations by measuring the amount of light they absorb (Bennett 1997). In this study, the nanoparticle used is iron(III) oxide nanoparticle, and thus, the wavelength of the light source used is $248.33 \mathrm{~nm}$. Before carry out this analysis, standard solutions are prepared in order to construct the calibration curve.

\section{Results and discussion}

\section{Porosity of the column}

After the construction of column and the sieving process of the sand were done, the column was packed with the sand and agitated to promote compaction of the sand. $250 \mathrm{~mL}$ of water was introduced into the column and the volume of water at the outlet of the column was collected and measured. This step was repeated three to four times until the volume of water collected at the outlet of the column was constant. The results are recorded in Table 1.

From the results obtained, the effective volume of pores, $V_{\mathrm{v}}$ is $395 \mathrm{~mL}$ which is equivalent to $395 \mathrm{~cm}^{3}$. The column constructed has a diameter of $6.6 \mathrm{~cm}$ and a length of $35 \mathrm{~cm}$. The volume of the column was calculated using the formula

$V_{\mathrm{T}}=\frac{\pi D^{2}}{4} \times l$

$V_{\mathrm{T}}=1197.42 \mathrm{~cm}^{3}$

The porosity of the column was calculated using the formula

$$
\begin{aligned}
& \varnothing=\frac{V_{\mathrm{v}}}{V_{\mathrm{T}}} \times 100 \\
& \varnothing=\frac{395}{1197.42} \times 100 \\
& \varnothing=32.99 \% .
\end{aligned}
$$

\section{Concentration effects of nanoparticles at different phase}

Atomic absorption spectroscopy (AAS) is an analytical technique that measures the concentration of the elements. In this study, AAS was used to measure the concentration of iron(III) oxide nanoparticles in two different solutions which were water and paraffin oil. There were 8 different samples analyzed by AAS, and the results of these samples were then compared and their differences will be discussed in detailed. Figure 2 shows the graph of concentration of iron(III) oxide nanoparticles in different solutions with pore volume ranging from 0.25 to $1.0 \mathrm{PV}$.

Based on the graph as shown in Fig. 2, it can be seen that the concentration of $\mathrm{Fe}_{2} \mathrm{O}_{3}$ in water is not constant as it has a sudden drop at $0.45 \mathrm{PV}$ and a sharp decrease in between 0.8 and 1.0 PV. Iron(III) oxide is sparingly soluble in water, and it tends to aggregate due to their large surface

Table 1 Volume of water introduced and collected

\begin{tabular}{llll}
\hline Trial & $\begin{array}{l}\text { Volume of water } \\
\text { introduced }(\mathrm{mL})\end{array}$ & $\begin{array}{l}\text { Volume of water } \\
\text { collected }(\mathrm{mL})\end{array}$ & $\begin{array}{l}\text { Volume of water } \\
\text { retained in column } \\
(\mathrm{mL})\end{array}$ \\
\hline 1 & 250 & 20 & 230 \\
2 & 250 & 155 & 95 \\
3 & 250 & 205 & 45 \\
4 & 250 & 220 & 30 \\
5 & 250 & 255 & -5 \\
6 & 250 & 250 & 0 \\
& & Total & 395 \\
\hline
\end{tabular}


Fig. 2 Graph of concentrations versus pore volume in water and paraffin oil at room temperature

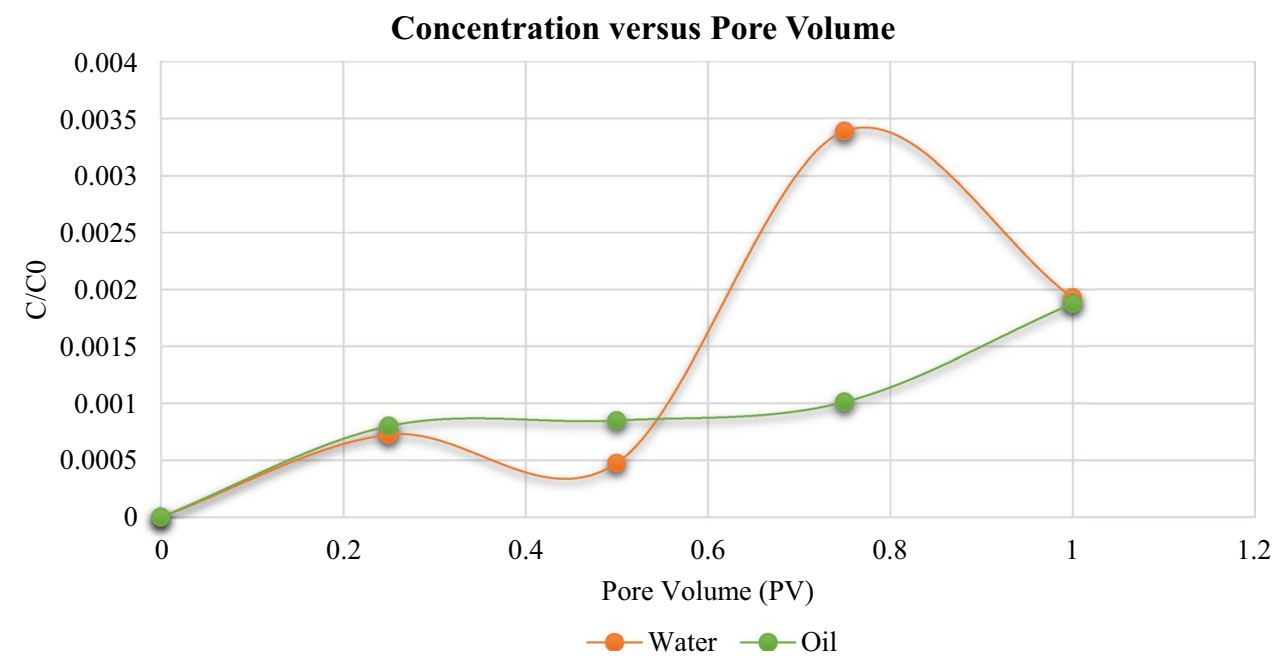

to volume ratio exhibiting strong superparamagnetic properties (Archarya et al. 2017). Although the nanofluid have been stirred using magnetic stirrer for $1 \mathrm{~h}$ and ultrasonicated for $6 \mathrm{~h}$, it was still not well-dispersed. At $0.25 \mathrm{PV}$, the nanoparticles injected into the column started to accumulate and clumped together as the particles were not mix well in the water. When the water flowed out from the column, the nanoparticles still accumulated and transported slowly in the column and therefore, the water at the exit did not contain much nanoparticles and resulted in a sudden drop at the outlet concentration from 0.25 to $0.45 \mathrm{PV}$. Starting from 0.5 $\mathrm{PV}$, the concentration of nanoparticles increased sharply due to the emergence of the accumulated nanoparticles. At 0.8 $\mathrm{PV}$, a sudden drop in the concentration value happened due to the same reason of accumulation of nanoparticles in the column. The phenomenon of sudden drop in concentration also can be explained by the narrowing of flow area and the differential pressure which lead to a velocity increase of the nanofluid when it flows from pores to throats. The small water molecules will tend to flow faster than the nanoparticles causing accumulation of NP at the pores (Sun 2017). Hence, less particles are collected at the outlet of the column and caused a sudden drop at outlet concentration.

Among all two graphs, the graph of oil showed a stable increased from 0.25 to $1.0 \mathrm{PV}$. From the graph shown in Fig. 2, it can be seen that nanoparticles were stable and flowed accordingly with oil along the column. The introduction of nanoparticles solution into oil tends to reduce the viscosity of oil and caused the oil to flow smoothly along the column. Unlike the other two conditions, the stable interaction of nanoparticles and oil in the column resulted in a graduated increased in outlet concentration of nanoparticles.

\section{The conductivity study on the effects of pore volume injected}

From the graph shown in Fig. 3, it can be seen that the conductivity increased with the pore volume injected into
Fig. 3 Graph of conductivity versus pore volume in water at room temperature

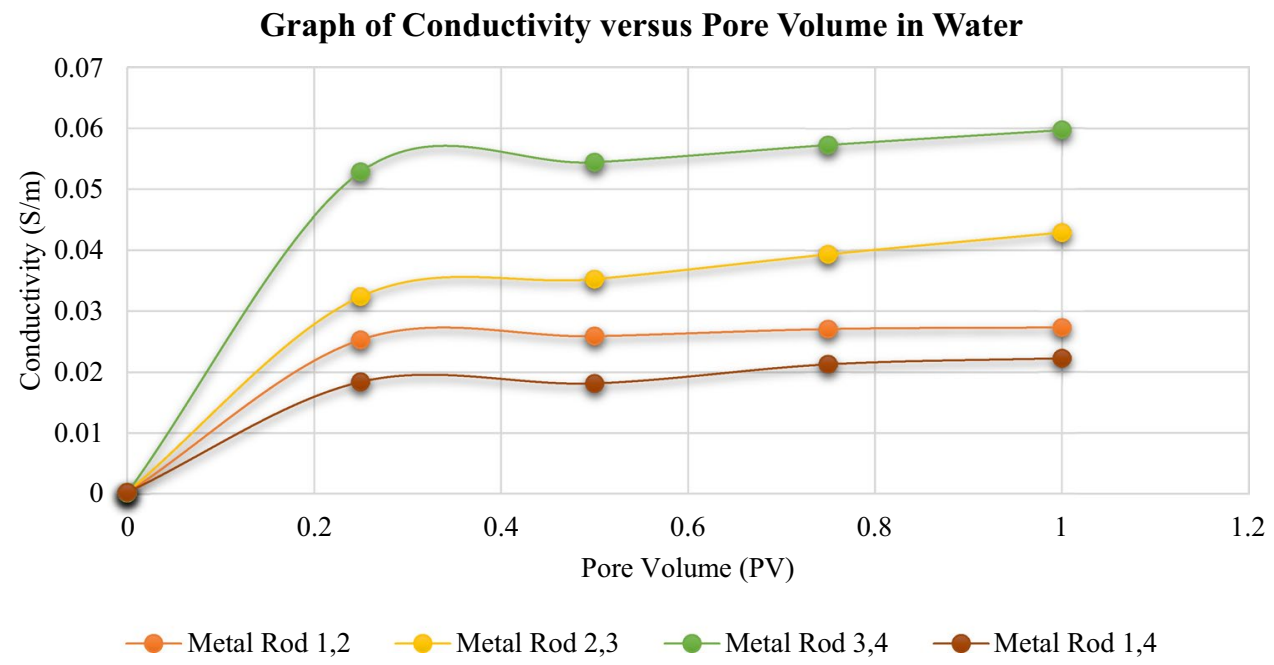


the column. In this experiment, the column was divided into three parts which represented by metal rod 1,2, metal rod 2,3 and metal rod 3.4, while metal rod 1,4 represented the entire column. At the first injection, the first section of the column will be saturated with nanoparticle solution before the excess nanofluid flowed to other part of the column. After second injection, the nanoparticle solution will push the nanoparticle solution in the first section to the next section of the column. This could explain the obvious increased of conductivity value in metal rod 2,3 , metal rod 3,4 and metal rod 1,4. Among all metal rods, metal rod 1,2 showed an almost constant conductivity reading of 0.025 $\mathrm{S} / \mathrm{m}$. This is because the conductivity reading at metal $\operatorname{rod} 1,2$ at the first injection $(0.25 \mathrm{PV})$ was already at the saturated condition of nanofluid. Hence, any new injection of nanoparticle solution does not affect the conductivity value measured at that particular section.

Temperature effect is not taken into consideration in this study; however, a higher temperature would cause a fluctuation in the conductivity readings due to the aggregation of the NP which would affect the transportation of the nanofluid in the porous media. The concentration of nanoparticles suspension used in this study is $80 \mathrm{mg} / \mathrm{L}$. A high NP concentration would cause the accumulation of the aggregated NP at the pores which would affect the transportation of the nanofluid across the column (Hendraningrat and Torsæter 2014). Zayid revealed that the size of nanoparticle agglomerate has a significant effect on the conductivity readings (Zayid 2014). More studies are essential to understand the effect of concentration on the transportation of oxide nanoparticles in porous media for the prediction of the role of oxide nanoparticles at different concentration for the enhancement of oil recovery.
Based on the graph shown in Fig. 4, it can be seen that the trend of the graph in paraffin oil was increasing with pore volume injected. However, the overall conductivity value in paraffin oil was higher than that in water. This is because paraffin oil is hydrocarbon with Van der Waals forces and contributed to positive conductivity readings (Göltl 2012). In addition to that, the nanoparticles had stable interaction with oil and thus no aggregation happened and more nanoparticles flowing in the column contributed to higher conductivity reading. In this study, the conductivity was measured by determining the resistance of the medium between two metal rods separated by a fixed distance. The conductivity is inversely proportional to resistance which indicated that a shorter distance between two metal rods resulted in a higher conductivity value. Metal rod 3,4 showed the highest conductivity readings compared to other metal rods. This could be explained by the shortest distance between metal rod 3 and 4 . The distance between metal rod 1 and 4 was the longest and thus, it had the lowest conductivity reading.

\section{The resistivity study on the effect of pore volume injected}

Based on Fig. 5 as shown above, it can be seen that at the first injection of $0.25 \mathrm{PV}$, the resistivity decreased from metal rod 1,4 to metal rod 1,2 , followed by metal rod 2,3 and metal rod 3,4. Among all metal rods, metal rod 1,4 showed the highest resistivity value. This could be explained by the longest distance between metal rod 1 and 4 . A longer distance indicated that the nanoparticles need to travelled further and the obstacles met during the transport were also increased and therefore, resistivity value increased. The resistivity of metal rod 1,4 showed a sudden decrease after
Fig. 4 Graph of conductivity versus pore volume in paraffin oil at room temperature

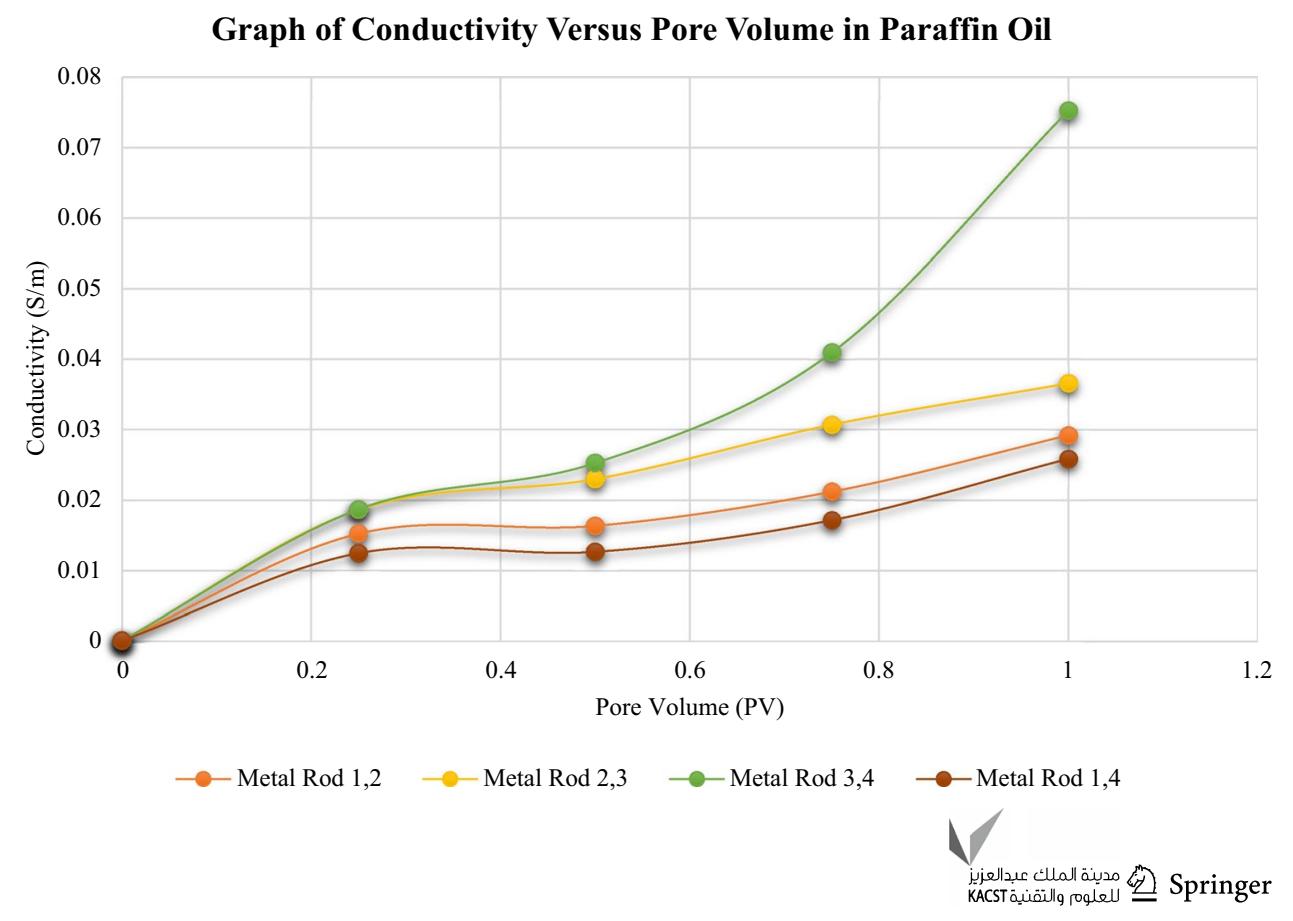


Fig. 5 Graph of resistivity versus pore volume in water at room temperature

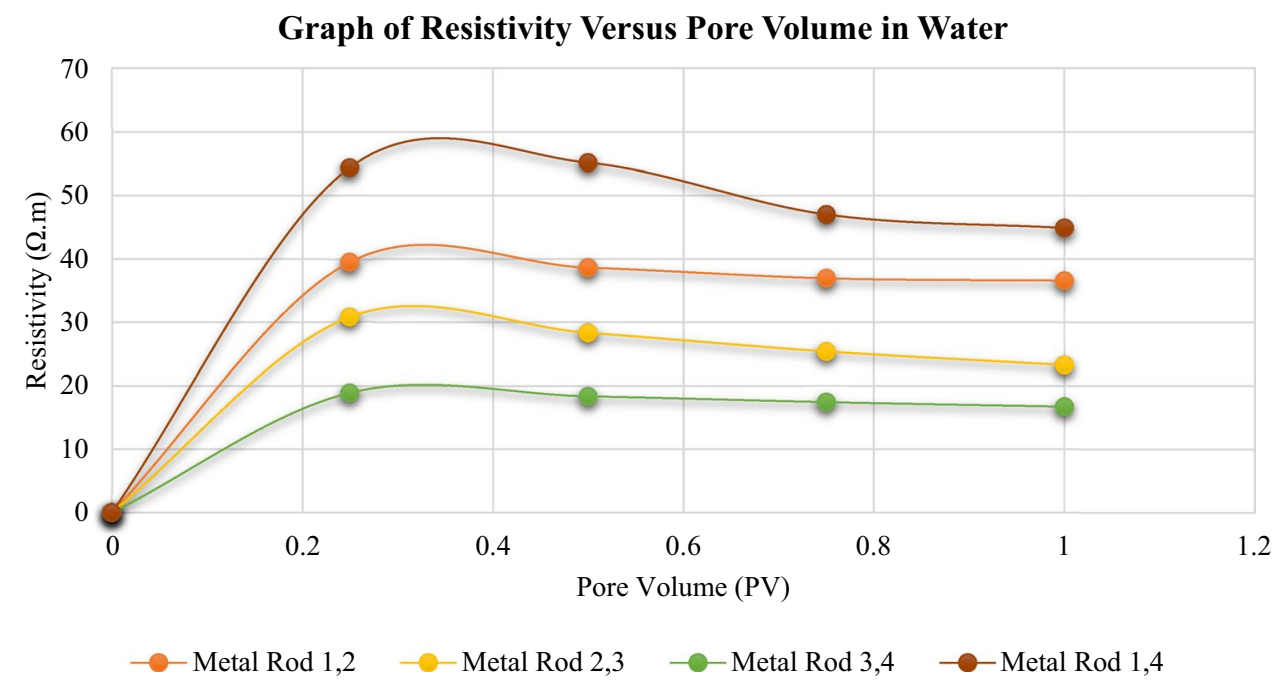

the injection of 0.5 PV. This is because after the second injection, the concentration of nanoparticle increased which resulted in the increased in conductivity. Thus, resistivity after $0.5 \mathrm{PV}$ injection reduced sharply.

The graph shown in Fig. 6 showed that the resistivity measured in paraffin oil decreased with the increased in pore volume injection. It can be seen that the resistivity measured in paraffin oil $(80.23 \Omega \mathrm{m})$ was the highest among all two graphs. This is because paraffin oil is an organic compound which has Van der Waals intermolecular forces and this will result in high conductivity value which indicated a low resistivity reading. Besides, paraffin oil had higher viscosity which became the resistance for the flow of nanoparticle solution. These were the reasons explaining why the resistivity in paraffin oil was higher than resistivity in water.

\section{Conclusion}

The objective of this experiment was to study the oxide nanoparticle transport in low-porosity sand pack and the distance transport by the nanoparticles in the absence and presence of oil. Based on the concentration graph, the graph of paraffin oil showed the most stable increased from 0.25 to $1.0 \mathrm{PV}$, whereas the graph of water showed significant fluctuations. Hence, it can be concluded that iron(III) oxide nanoparticles flowed smoothly in paraffin oil, followed by water. Among the two samples, water showed the lowest conductivity reading and highest resistivity value which were $0.022 \mathrm{~S} / \mathrm{m}$ and $44.90 \Omega \mathrm{m}$, respectively, after $1.0 \mathrm{PV}$ injection. In the presence of paraffin oil, the conductivity and resistivity reading were $0.026 \mathrm{~S} / \mathrm{m}$ and $38.66 \Omega \mathrm{m}$,
Fig. 6 Graph of resistivity versus pore volume in paraffin oil at room temperature

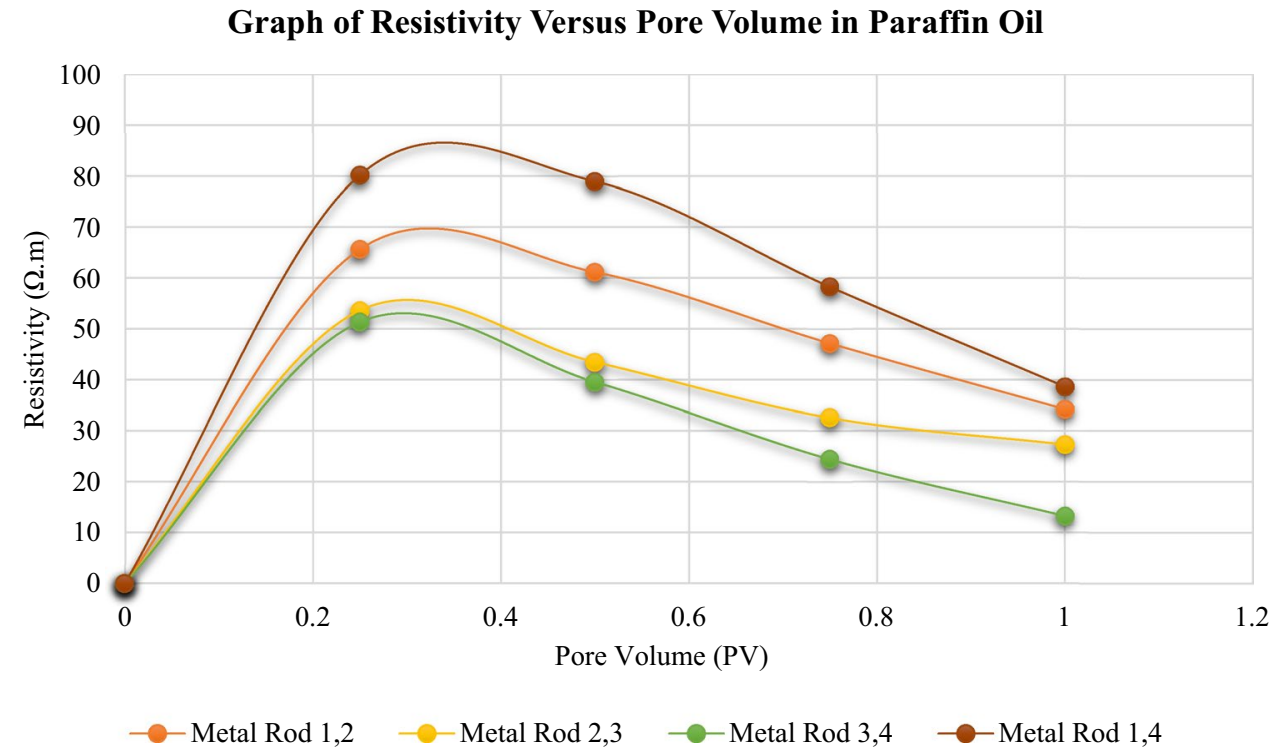


respectively. This indicated that the transport of nanoparticles in the presence of oil had lower resistance compared to the resistance in the absence of oil. Thus, it can be concluded that the distance transported by nanoparticles in the presence of oil were longer compared to the distance transported in the absence of oil.

Acknowledgements Thanks to Faculty of Chemical and Natural Resources Engineering Universiti Malaysia Pahang and Research and Innovation center for giving the research Grant RDU180325 for financial support.

Open Access This article is distributed under the terms of the Creative Commons Attribution 4.0 International License (http://creativeco mmons.org/licenses/by/4.0/), which permits unrestricted use, distribution, and reproduction in any medium, provided you give appropriate credit to the original author(s) and the source, provide a link to the Creative Commons license, and indicate if changes were made.

\section{References}

Agista MG (2018) A state-of-the-art review of nanoparticles application in petroleum with a focus on enhanced oil recovery. Appl Sci 8:871

Archarya G, Mitra AK, Cholkar K (2017) Chapter 10 - Nanosystems for diagnostic imaging, biodetectors, and biosensors. In: Mitra AK, Cholkar K, Mandal A (eds) Emerging nanotechnologies for diagnostics, drug delivery and medical devices. Micro and nano technologies. Elsevier, Boston, pp 217-248

Bayat A (2015) Transport and retention of engineered $\mathrm{Al}_{2} \mathrm{O}_{3}, \mathrm{TiO}_{2}$, and $\mathrm{SiO}_{2}$ nanoparticles through various sedimentary rocks. Sci Rep 5:14264
Bennett P (1997) Introducing atomic absorption analysis. Varian Australia Pty Ltd, Mulgrave

Bradford S (2012) Facilitated transport of copper with hydroxyapatite nanoparticles in saturated sand. Soil Chem 76:375-388

Caldelas FM (2011) Factors governing distance of nanoparticle propagation in porous media. Society of Petroleum Engineers, New York

Göltl FG (2012) Van der Waals interactions between hydrocarbon molecules and zeolites. J Chem Phys 137:114111

Hendraningrat L, Torsæter O (2014) Unlocking the potential of metal oxides nanoparticles to enhance the oil recovery. In: Proceedings of the annual offshore technology conference-Asia, Kuala Lumpur, Malaysia. https://doi.org/10.2118/24696-MS

Hoet P (2004) Nanoparticles-known and unknown health risks. J Nanobiotechnol 2:2-12

Jeong S (2009) Aggregation and transport of copper oxide nanoparticles in porous media. J Environ Monit 11:1595-1600

Sun XZ (2017) Application of nanoparticles in enhanced oil recovery: a critical review of recent progress. Energies 10:345

Wang C (2012) Retention and transport of silica nanoparticles in saturated porous media: effect of concentration and particle size. Environ Sci Technol 46:7151-7158

Zayid A (2014) Factors efecting the electrical conductivity and zeta potential of alumina nanoluids. Master Thesis, Dalhousie University, Halifax, Nova Scotia

Zettner A (1964) Principles and applications of atomic absorption spectroscopy. Adv Clin Chem 7:1-62

Publisher's Note Springer Nature remains neutral with regard to jurisdictional claims in published maps and institutional affiliations. 